\title{
Knowledge Base Copyright Law - Wissensbank Urheberrecht für alle?
}

Preprint, erscheint als Beitrag in: Schweighofer, E. et.al. (Hrsg.), Aktuelle Fragen der Rechtsinformatik - Effizienz von e-Lösungen in Staat und Gesellschaft (IRIS 2005), Verlag Boorberg, 2005, 351-358.

\section{Peter Trybus}

Wirtschaftsuniversität Wien, Institut für Bürgerliches Recht, Handels- und Wertpapierrecht, Abteilung für Informationsrecht und Immaterialgüterrecht

Althanstraße 39-45, Stiege 5, 1. Stock, A-1090 Wien

peter.trybus@wu-wien.ac.at

\section{Michael Nentwich}

Österreichische Akademie der Wissenschaften, Institut für Technikfolgen-Abschätzung Strohgasse 45/5, A-1030 Wien

mnent@oeaw.ac.at

Schlagworte: Urheberrecht, Datenbank, Open Content

Abstract: $\quad$ Das Projekt „Knowledge Base Copyright Law“ (KB:LAW|C) bezweckt die Entwicklung eines innovativen Datenbanksystems sowie die Erstellung der Inhalte dieser Datenbank, die sich mit Urheberrecht und dessen praktischen Auswirkungen auf die Creative Industries beschäftigen. Die Knowledge Base soll diese Informationen sowohl für Laien als auch Experten zugänglich machen, indem neben der Grundinformation stets auch eine umfassende rechtliche Analyse zur Verfügung stehen wird. Die Fragen sollen in verschiedenen Sprachen und aus der Sicht verschiedener Länder und deren Rechtssystemen beantwortet werden, was bei urheberrechtlichen Problemstellungen im digitalen Umfeld eine notwendige, aber bisher nicht verwirklichte Funktionalität darstellt. Urheberrechtswissen und dessen Anwendung soll so auch außerhalb der Wissenschaft und Forschung vermittelt werden.

\section{Ausgangslage}

\subsection{Projekt Creative Access}

Im Rahmen des vom Programm „SciENCE for Creative Industries“ des Wiener Wissenschafts-, Forschungs- und Technologiefonds 
$(\text { WWTF })^{1}$ geförderten Projekts „Creative Access: Digital Archives between Open Knowledge Society and Commodification in Vienna" entsteht in einem der Arbeitskreise in Zusammenarbeit zwischen dem Demokratiezentrum Wien ${ }^{2}$, dem Instituts für TechnikfolgenAbschätzung (ITA) der Akademie der Wissenschaften ${ }^{3}$ und der Abteilung für Informationsrecht und Immaterialgüterrecht an der Wirtschaftsuniversität Wien ${ }^{4}$ eine Wissensbank zu urheberrechtlichen Fragestellungen mit Fokus auf den Themenkreis der digitalen Archive. ${ }^{5}$ Ziel ist es durch diese Datenbank und die damit vermittelten juristischen Grundlagen die Creative Industries und deren Entwicklung zu fördern. ${ }^{6}$

\subsection{Urheberechtliche Problemstellungen der Creative Industries im Lichte der Digitalisierung}

Die Klärung urheberechtlicher Problemstellungen ist insbesondere im Zeitalter der Informationsgesellschaft und der damit einhergehenden Digitalisierung von Werken eines der zentralen Anliegen der Creative Industries und der Gesellschaft. Die Produkte der Creative Industries werden zunehmend (auch) digital erfasst, gesammelt und vertrieben. Werke werden immer häufiger über OnlineArchive zugänglich gemacht, was nicht nur technische, organisatorische und ökonomische Fragen aufwirft, sondern auch zahlreiche juristische, insbesondere urheberrechtliche Problemstellungen schafft. Das Ineinandergreifen zahlreicher nationaler, europäischer und internationaler Normen, ${ }^{7}$ die große

\footnotetext{
${ }^{1}$ http://www.wwtf.at/

${ }^{2}$ http://www.demokratiezentrum.org/.

${ }^{3}$ http://www.oeaw.ac.at/ita/.

${ }^{4} \mathrm{http}: / / \mathrm{www}$.infolaw.at/.

${ }^{5}$ Weitere Informationen zur Knowledge Base Copyright Law finden sich unter http://www.kb-law.info.

${ }^{6}$ Unter den Begriff der Creative Industries lässt sich eine breite Palette an Tätigkeiten und Unternehmenszweigen subsumieren. Sie haben ihren Ursprung in der individuellen Kreativität, im künstlerischen Schaffen, im Geschick und Talent und tragen durch das Schaffen und die Verwertung von geistigem Eigentum zum Wohlstand der Gesellschaft bei. Siehe auch http://www.britishcouncil.org/artscreative-industries-definition.htm (13.4.2005) sowie http://en.wikipedia.org/wiki/ Creative_industries (13.4.2005).

${ }^{7}$ Exemplarisch kann das Zusammenspiel des Welturheberrechtsabkommen in der Pariser Fassung vom 24.7.1971, BGBI 1982/293, der Richtlinie 2001/29/EG des Europäischen Parlaments und des Rates vom 22.5.2001 zur Harmonisierung bestimmter Aspekte des Urheberrechtes und der verwandten Schutzrechte in der
} 
Kasuistik der Rechtsprechung und die immer häufiger werdenden länderübergreifenden Sachverhalte machen das Urheberrecht zu einer äußerst komplexen Materie. Diese ist für juristische Laien nur sehr schwer durchdringbar und überblickbar, weshalb der Bedarf nach der Vermittlung von Urheberrechtswissen unter Nicht-Juristen besonders groß ist.

\subsection{Online Rechtsinformationssysteme und deren charakteristische Defizite}

Zunächst wurde ein extensives Screening einschließlich einer Analyse des Stands der internationalen Forschung im Bereich der Online-Datenbanksysteme, die rechtliche Informationen sowohl für Laien als auch Experten zur Verfügung stellen, durchgeführt. Die Befundnahme ergab, dass zwar zahlreiche urheberechtliche Informationen - meist als Sammlungen von „Frequently Asked Questions" (FAQ) - online verfügbar sind, diese jedoch die folgenden wesentlichen Funktionalitäten nicht zufrieden stellend erfüllen:

- Informationsqualität und umfassende Abdeckung des Themengebiets: Die derzeit auf juristische Laien ausgerichteten, online verfügbaren Informationsangebote bieten nur einen sehr oberflächlichen Überblick über urheberrechtliche Problemstellungen und decken meist nur einen sehr beschränkten Teilbereich $a b .^{8}$ Deshalb können diese Informationsquellen den Nutzern nur selten die tatsächlich gesuchten Antworten und Information zur Verfügung stellen.

- Qualität des Informationszugangs (innovativer, user-orientierter Zugriff auf relevante Daten): Bisher gibt es in diesem Bereich kein System, welches den Nutzern erlaubt, die in einer bestimmten Situation benötigten Informationen gezielt abzurufen. ${ }^{9}$ Dadurch ist es nicht möglich, auf bestehende Informationen rasch zuzugreifen und diese sinnvoll anzuwenden. Vielmehr sind Nutzer bisher gezwungen, zunächst eine große Datenmenge zu durchforsten, bevor die gewünschte Information gefunden wird. Oftmals wird erst nach einem aufwändigen Suchprozess sichtbar, dass die

Informationsgesellschaft und des Bundesgesetz über das Urheberrecht an Werken der Literatur und der Kunst und über verwandte Schutzrechte, BGBI. 111/1936 idF BGBI I 36/2003, genannt werden.

${ }^{8}$ Siehe zB http://www.copyright.gov/help/faq/ (9.4.2005).

${ }^{9}$ Siehe zB http://remus.jura.uni-sb.de/urheberrecht/index.html (9.4.2005). 
gewünschte urheberrechtliche Hilfestellung in der Informationsquelle überhaupt nicht enthalten ist.

- Qualität und Aufbereitung der rechtlichen Informationen: Einer der wesentlichen Mängel von Rechtsinformationssystemen in diesem Bereich ist, dass es kein Informationsangebot gibt, welches urheberechtliche Information sowohl überblicksartig, in einer für (juristische) Laien verständlichen Form darstellt, als auch umfassende rechtliche Analysen anbietet, wenn der User sich entscheidet, in die Tiefe $\mathrm{zu}$ gehen und profunde rechtliche Informationen in Anspruch zu nehmen. ${ }^{10}$

\section{Multidimensionaler Lösungsansatz und Konzept der KB:LAW|C}

\subsection{Zwei Ebenen: Laien- und Expertenantwort}

Schon beim Erstellen und Evaluieren des Konzepts der Wissensbank zeichnete sich ab, dass die Probleme beim Vermitteln juristischer Informationen an Laien nur mittels unterschiedlicher Ebenen der Informationsdichte gelöst werden können. Dem User muss zunächst ermöglicht werden, auf eine einfache Frage, eine kurze, für juristische Laien verständliche Antwort zu erhalten, die das Grundkonzept erörtert und die Lösung der Fragestellung überblicksartig darstellt. Dies soll dem Betrachter eine erste Orientierungshilfe bieten, sodass deutlich erkennbar ist, ob das konkrete Anliegen durch die Fragestellung erfasst ist und inwieweit es durch die Antwort lösbar ist. Dies genügt bei urheberechtlichen Problemstellungen allerdings nur selten, weil zur weiteren Klärung meist detaillierte juristische Fachinformation erforderlich ist. Deshalb wird dem Nutzer auf einer zweiten Ebene eine fundierte juristische Analyse zur Verfügung gestellt, die sowohl den interessierten Laien als auch den juristischen Experten gleichermaßen ansprechen soll. Beide Antwortebenen sind mit zahlreichen Zitaten und Querverweisen zu versehen. ${ }^{11}$ Die Beantwortung von Fragestellungen auf zwei

\footnotetext{
10 Der Großteil der verfügbaren Information beantwortet die Fragestellungen ausschließlich überblicksartig und ohne juristische Detailerörterung, siehe zB http://www.oeaw.ac.at/personalwesen/e-urh_FAQ/ (9.4.2005), http://www.bmbwk.gv.at/medienpool/10109/FAQ-Sammlung.pdf (9.4.2005).

${ }^{11} \mathrm{Zu}$ den Zugangsmöglichkeiten und zur Navigation innerhalb der Datenbank siehe Abschnitt 2.4 .
} 
Ebenen stellt somit einen neuen Lösungsansatz für das Vermitteln komplexer juristischer Materien dar.

\subsection{Territoriale Dimension}

Die Globalisierung sowie die zunehmende Digitalisierung von Werken bewirken immer öfter länderübergreifende Sachverhalte, die eine vergleichende Betrachtung der unterschiedlichen Rechtssysteme und deren Regelungen im Bereich des Urheberechts erfordern. Deshalb ermöglicht das User-Interface von KB:LAW|C den Wechsel von der Rechtslage eines Landes in die eines anderen, wodurch dem Nutzer zu ein und derselben Frage Antworten aus der Sicht unterschiedlicher Rechtssysteme zur Verfügung stehen. ${ }^{12}$

\subsection{Mehrsprachigkeit}

Eine weitere Hürde, die hier überwunden werden soll, ist die Sprachbarriere. Die Datenbank wurde so gestaltet, dass alle Antworten in verschiedenen Sprachversionen erfasst und verfügbar gemacht werden können. In der ersten Ausbaustufe wird der urheberrechtliche Content aus Kapazitätsgründen nur auf Englisch und der Sprache des Landes, auf dessen Rechtslage Bezug genommen wird, erstellt. Die $K B: L A W \mid \subset$ wird nicht nur über das Internet für die Öffentlichkeit frei zugänglich sein, sondern soll durch die multilinguale Ausrichtung auch gewährleisten, dass möglichst viele User die Inhalte tatsächlich verstehen und anwenden können. Eine Frage und die dazugehörigen Antworten können demnach prinzipiell in jeder erdenklichen Sprache erfasst werden. Dies stellt die Grundlage für eine Ausweitung des Informationsangebots auf zahlreiche weitere Länder dar.

\subsection{Multiple Zugangsmöglichkeiten}

Der Zugriff auf $\mathrm{KB}: L A W \mid \subset$ ist von Zeit und Ort unabhängig. Nicht nur der Abruf der Informationen ist jederzeit online möglich, auch die Eingabe der Inhalte in die Datenbank erfolgt online über das eigens entwickelte Input-Interface, welches den Autoren eine möglichst flexible Erfassung und Editierung der Beiträge ermöglicht. Dieses Konzept schafft somit auch ideale Voraussetzungen für die kooperative Erstellung des Contents innerhalb eines internationalen

${ }^{12}$ Dies gilt wiederum für beide Ebene der Beantwortung, die Laienantwort und die Expertenantwort. 
Netzwerks, in welchem Autoren aus verschiedenen Ländern Inhalte erstellen und bearbeiten können.

Um den Erfolg und die Akzeptanz der Datenbank zu gewährleisten, wird den Usern die Möglichkeit geboten, über verschiedene Zugangswege zur gewünschten rechtlichen Information zu gelangen. Nutzer können Abfragen entweder ausgehend von ihrer Profession, mittels intelligentem Schlagwort- und Volltextsuchmechanismus, einem Glossar oder thematisch untergliederter Frequently Asked Questions modifizieren. Insbesondere die Bereicherung der Suchfunktionalitäten um einen berufsgruppen-spezifischen Einstieg stellt eine wichtige Neuerung dar und wird den Nutzern helfen, die für sie relevanten Daten noch schneller zu finden.

Das Beschlagwortungssystem ist eine weitere wichtige Vorraussetzung für die Benutzerfreundlichkeit des User-Interface. Über die Option „verwandte Dokumente" ermöglicht es den Zugriff auf all jene Dokumente in der Wissensbank, welche thematisch mit dem Inhalt des aktuellen Dokuments in Verbindung stehen. Dies gestattet einen direkten, intuitiven Wechsel zwischen zueinander verwandten Fragestellungen, ohne dass zusätzliche Zwischenschritte oder Suchabfragen erforderlich sind.

\section{Inhalte der Datenbank}

1. Frequently Asked Questions als Ankerpunkt: Eine zentrale Rolle in der Informationsvermittlung der Datenbank kommt den FAQs zu. Die einfach formulierten und für Laien verständlichen Fragen wurden mit Hilfe von Treffen mit Vertretern der Creative Industries sowie eines Online Fragebogens gesammelt, evaluiert und zu einem umfassenden Fragenkatalog zusammengestellt. ${ }^{13}$ Ein wesentlicher Teil dieser Arbeit beschäftigte sich mit der Gestaltung und Formulierung der urheberechtlichen Fragestellungen, damit diese juristische Begrifflichkeiten in einer für Laien zugänglichen Form ausdrücken.

2. Laienantwort und Expertenantwort: Ein Team von Urheberechtsexperten widmet sich der Beantwortung der Fragen auf zwei verschiednen Ebenen. Die Methodik und das Konzept wird in Abschnitt 2.1. näher beschrieben.

3. Referenzierte Quellen: Neben den Fragen und den dazugehörigen Antworttexten wird die Datenbank auch die

\footnotetext{
13 Die über 100 spezifisch formulierten Fragen werden laufend überarbeitet und ergänzt, sodass diesbezügliche Vorschläge über das Online-Formular (http://www.kb-law.info/copyright/) gerne entgegen genommen werden.
} 
referenzierten Quellen enthalten, welche an den entsprechenden Zitatstellen direkt verlinkt und abrufbar sind. Der Nutzer kann so auf die Volltexte der Rechtsquellen, Entscheidungen und - in einem beschränkten Maß auch - Literatur ${ }^{14}$ zugreifen, ohne diese aufwändig selbst suchen zu müssen.

4. Metadaten und Glossar: Damit die Datenbank und insbesondere die zahlreichen Querverbindungen wie beschrieben funktionieren werden bei der Eingabe über das selbst konzipierte Input-Interface zahlreiche Metadaten ${ }^{15}$ erfasst und verarbeitet. Zusätzlich wird ein Glossar erstellt, welches Grundbegriffe und -konzepte erklärt und den Nutzern wiederum Querverbindungen $\mathrm{zu}$ all jenen Dokumenten anbietet, die sich auf diesen Begriff beziehen.

\section{Technische Komponenten}

1. Datenbankstruktur: Um der Multidimensionalität gerecht zu werden, musste eine komplexe Datenbankstruktur völlig neu konzipiert und entwickelt werden.

2. Dateneingabe: Die Dateneingabe wurde als webbasiertes System konzipiert und bietet den Autoren eine einfach zu bedienende Oberfläche im Registerkarten-Format. Der eigens angepasste OnlineEditor bietet die notwendigen Textverarbeitungsfunktionen und ermöglicht Verlinkungen zu anderen Datenbankelementen sowie die Erstellung von Zitaten innerhalb eines Dokuments.

3. User-Interface: Um die Suche nach und den Abruf von Informationen möglichst intuitiv und benutzerfreundlich zu gestalten, wird ein User-Interface in Form einer übersichtlichen, auf einem Bildschirm erfassbaren Website gestaltet. ${ }^{16}$ Das Ziel ist es, stets ein direktes Umsteigen zwischen der Rechtslage verschiedener Länder, den unterschiedlichen Sprachfassungen, der Laien- und Expertenebene sowie den Antworten und der Dokumentation (Rechtsquellen, Entscheidungen, Literatur) zu ermöglichen.

4. Implementierung mit Open Source Produkten: Die genannten technischen Komponenten waren mit bestehenden Produkten nicht in der gewünschten, sehr anspruchsvollen Form umsetzbar, weshalb

\footnotetext{
${ }^{14}$ Mangels Verfügbarkeit in digitaler Form und der fehlenden finanziellen Mittel für eine Lizenzierung kann zitierte Literatur nicht selbst in der Datenbank erfasst werden. Sofern online verfügbar, wird ein Link zu dem entsprechenden Dokument angeboten.

${ }^{15}$ Hier haben neben den Informationen zu Autor, Titel, etc. insbesondere die thematischen sowie zielgruppenspezifischen Schlagwörter eine wichtige Funktion.

${ }^{16}$ Die Arbeiten am User-Interface sind noch nicht abgeschlossen.
} 
diese im Rahmen des Projekts unter der Verwendung von OpenSource-Produkten programmiert wurden. Folgende Software wurde eingesetzt: $\mathrm{PHP}^{17}$, PostgreSQL ${ }^{18}, \mathrm{ADOdb}^{19}$ und Smarty Template Engine $^{20}$. Die ausschlaggebenden Gründe für den Einsatz von OpenSource-Software waren vor allem der Kostenfaktor, die einfache Portabilität auf andere Systeme sowie die Förderung von Open Content.

\section{Ziele und Ausblick}

Kurzfristig wird die Datenbank für den primär gedachten Einsatzbereich „Urheberrecht in der Informationsgesellschaft“ optimiert werden. In der jetzt laufenden Pilotphase können nur exemplarische Fragen und Antworten und nur für eine Handvoll Länder gegeben werden. Unser Ziel ist es, die finanziellen Grundlagen für ein juristisches Projektteam in Österreich sicherzustellen und in weiterer Folge ein internationales Netzwerk an Korrespondenten aufzubauen, welche weitere Inhalte, insbesondere aus der Sicht der Rechtslage des jeweiligen Landes, beitragen sollen. Da der Input in die Datenbank zur Gänze webbasiert ist, bestehen keine örtlichen Schranken für das Erstellen, Hinzufügen und Bearbeiten weiterer Artikel und der dazugehörigen Dokumentation.

Mittelfristig könnte $\mathrm{KB}: L A W \mid \subset$ generell alle Fragen des Urheberrechts abdecken. Mit voranschreitender Erstellung und Erfassung der Inhalte hat die Knowledge Base das Potential, zu einem gängigen Werkzeug im Umgang mit urheberrechtlichen Fragestellungen zu werden.

Längerfristig gedacht, könnte das Projekt KB:LAW über die Pilotanwendung im Bereich Urheberrecht hinaus Bedeutung erlangen. Die von uns gemachten Erfahrungen bei der Strukturierung und Aufbereitung von Rechtswissen könnten für weitere derartige Projekte Pate stehen. Insbesondere könnte die Applikation in Zukunft auch für andere Rechtsbereiche eingesetzt werden, weil die Datenbankfunktionalitäten und die erfassbaren Inhalte in gleicher Weise ideal geeignet erscheinen.

\footnotetext{
${ }^{17}$ Skriptsprache für das Ein- und Ausgabe-Interface; siehe http://www.php.net/.

${ }^{18}$ Datenbankapplikation; siehe http://www.postgresql.net/.

19 Dient als Database Abstraction Layer, welcher die einfache Portierung der Applikation auf andere Datenbankserver gewährleistet; siehe http://adodb.sourceforge.net/.

${ }^{20}$ Ermöglicht die Trennung von Datenbankdesign und Applikationslogik; siehe http://smarty.php.net/.
} 